\title{
Soil-tool interaction of a simple tillage tool in sand
}

\author{
Lucian V. Fechete-Tutunaru ${ }^{1 *}$, Ferenc Gaspar ${ }^{1}$, and Zoltan Gyorgy ${ }^{2}$ \\ ${ }^{1}$ Technical University of Cluj-Napoca, Automotive Engineering and Transport Dept., B-dul Muncii 103-105 Cluj-Napoca, Romania \\ 2 INMA Bucharest, B-dul Ion Ionescu de la Brad, no. 6, Romania
}

\begin{abstract}
This study was focused to experimentally evaluate the draft force on a simple tillage tool operating in different conditions in sand. The soil-tool interaction was evaluated in controlled laboratory conditions in a soil bin. A full multilevel factorial experiment was used with one response variable and four experimental factors. The selected design 3 tools $\times 3$ tillage depth $\times 3$ rake angle $\times 3$ forward speed with two replications had a total of 243 runs. Influence of four independent variables including tillage depth (15, $20,25 \mathrm{~cm})$, rake angle $(25,35,50$ degrees $)$, forward speed $(0.67,0.98,1.39 \mathrm{~m} / \mathrm{s})$ and cutting-edge angle of the tool (30, 45 and 60 degrees) on draft force as the dependent variable was evaluated.
\end{abstract}

\section{Introduction}

Tillage, as a fundamental mechanical soil preparation phase, is an important operation in agriculture for crop production. It is a necessary preparation of land to improve soil characteristics for efficient plant growth [1]. In crop production soil tillage is related to significant energy consumption causing economic and environmental challenges. The energy demand for tillage is influenced by the tool design and configuration [2], soil properties and operating conditions [3-5]. An agreeable method to evaluate a given tillage tool energy requirement is determination of the draft force in different conditions [6].

Draft is a significant parameter for evaluation of tillage performance, efficiency and so the energy demand. Measurement and evaluation of draft in different conditions has been an important topic for research in this field [7]. In the last decades different soil bin facilities where developed to experimentally investigate soil-tool interactions and draft [8-10], to validate computational simulations on draft predictions $[1,11]$ and for development of optimized tillage implements [12].

Experimental studies on this topic conducted for different tillage tools and soil type show an increase of draft with the increase of tillage tool depth, forward speed and rake angle (lift angle) [1, 4-6, 13-15].

This study was focused to evaluate the influence of different parameters (forward speed, tillage tool depth, tool rake angle and tool cutting edge angle) on draft force. Experimental tests were conducted in laboratory conditions for a simple tillage tool in a fine and medium sand mixture. Obtained data's have been statistically analysed and explained.

\section{Materials and methods}

Three tillage tools models (tines), designated T30, T45 and T60 after their cutting-edge angle, were used in this comparative study. The tools are made from polypropylene PP-C, a material used for its good mechanical and chemical properties and easy machining. Between the mechanical properties of this material can be enumerated: tensile strength $32 \mathrm{MPa}$, flexural strength $41 \mathrm{MPa}$, and density $900 \mathrm{~kg} / \mathrm{m}^{3}$. The models used have dimensions $100 \times 100 \times 10 \mathrm{~mm}$ and were bevelled on the cutting edge at an angle of 30, 45 and 60 degrees respectively.

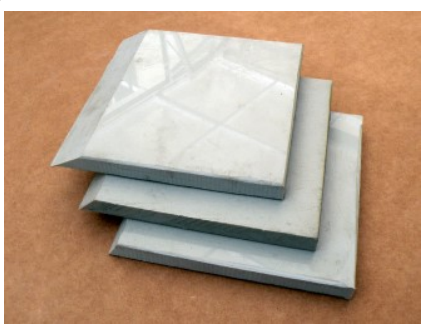

Fig. 1 Model tools with a cutting-edge angle of 30, 45 and 60 degrees

Experiments were conducted in controlled conditions in a soil bin at Automotive and Transportation Department, Faculty of Mechanical Engineering, Technical University of Cluj-Napoca, Romania.

Testing equipment consist in an indoor soil bin in which the tools can be moved on a circular trajectory with a diameter between $1700-2000 \mathrm{~mm}$ at a $900 \mathrm{~mm}$ maximum depth. Variable tools speed is assured by an electric motor $(3.2 \mathrm{~kW}, 720 \mathrm{rpm})$ and a dual system consisting in a gear reducer and belt driven transmission and a variable speed controller (Unidrive M200, 15kW). The equipment is completed with a DAQ system (HBM Spider 8) and different force transducers for measuring

\footnotetext{
* Corresponding author: lucian.fechete $@$ auto.utcluj.ro
} 
draft force. An overview of the soil bin is presented in fig. 2 .

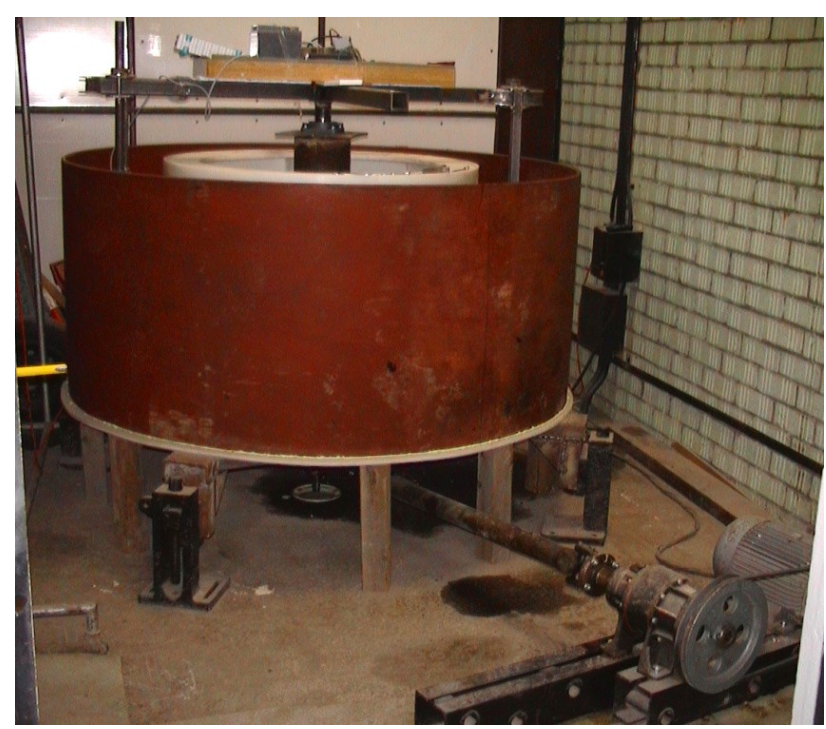

Fig. 2. Soil bin assembly.

The tool mounting frame fig. 3, allow the possibility of measuring draft force and offers several possibilities for adjusting angular and vertical position of the tool.

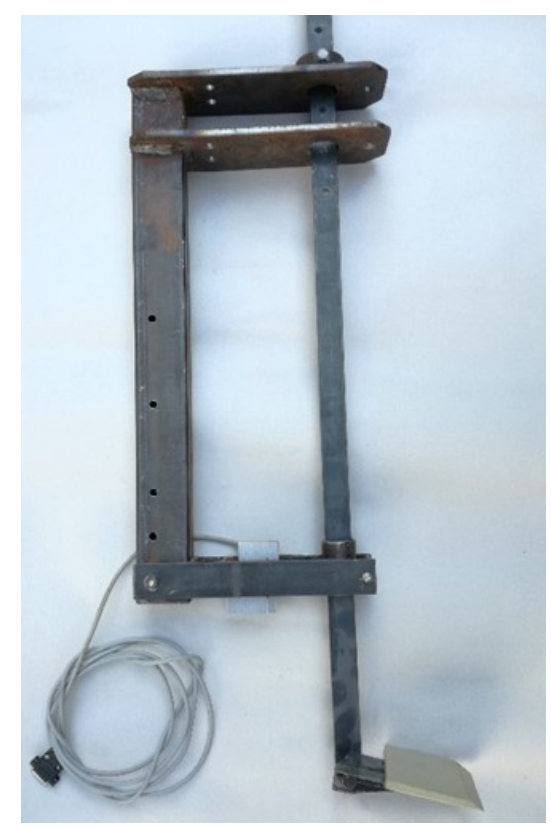

Fig. 3 Tool frame assembly.

In order to reduce as much as possible the influence of different physical parameters which characterize the agricultural soil, washed and sorted quartz sand was used. Particles diameter is between $0-0.3 \mathrm{~mm}$ which correspond to fine and medium sand according to ISO 14688. This way, the selected soil corresponds to a friction medium without cohesion and without structure. The decision was based on the affirmation , It should be noted that little data is available for purely cohesive soils or for very loose frictional soils" [16].

Soil penetration resistance (cone index) was measured and the results are presented in Table 1.
The three tools were evaluated for draft force individually in the soil bin. After the mounting of the individual tool on the frame, it was installed inside the bin at three depths and with three rake angles. Three different speed were also used. Each tool was dragged for a minimum one minute and the DAQ system constantly recorded the force with a rate of 50 measurements/sec.

Table 1. Soil penetration resistance of the selected soil [N].

\begin{tabular}{cccccc}
\hline No. & \multicolumn{5}{c}{ depth [mm] } \\
\hline \multicolumn{1}{c}{$\mathbf{5 0}$} & $\mathbf{1 0 0}$ & $\mathbf{1 5 0}$ & $\mathbf{2 0 0}$ & $\mathbf{2 5 0}$ \\
\hline $\mathbf{2}$ & 117 & 125 & 320 & 345 & 300 \\
\hline $\mathbf{3}$ & 100 & 205 & 240 & 336 & 350 \\
\hline $\mathbf{4}$ & 95 & 205 & 220 & 360 & 400 \\
\hline $\mathbf{5}$ & 100 & 210 & 270 & 350 & 295 \\
\hline Average & 102.4 & 191.0 & 258.0 & 322.2 & 333.0 \\
\hline Dispersion & $8.2 \%$ & $19.4 \%$ & $15.1 \%$ & $17.9 \%$ & $12.9 \%$ \\
\hline $\begin{array}{c}\text { Cone } \\
\text { parameters }\end{array}$ & Cone no. 4; cone base diameter $-25.33 \mathrm{~mm} ;$ \\
$\begin{array}{c}\text { Soil } \\
\text { resistance } \\
{\left[\mathbf{N} / \mathbf{c m}^{2}\right]}\end{array}$ & 20.48 & 38.20 & 51.60 & 64.44 & 66.60 \\
\hline
\end{tabular}

The factorial experiment $3 \times 3 \times 3 \times 3$ was run three times. The recorded data were processed with HBM Catman Easy and they were statistically analysed with Statgraphics Centurion XVII and Microsoft Excel.

\section{Results and discussion}

In this study, a full multilevel factorial experiment was used with one response variable and four experimental factors. The selected design 3 tools $\times 3$ tillage depth $\times 3$ rake angle $\times 3$ forward speed with two replications has a total of 243 runs.

Influence of four independent variables including tillage depth $(15,20,25 \mathrm{~cm})$, rake angle $(25,35,50$ degrees), forward speed $(0.67,0.98,1.39 \mathrm{~m} / \mathrm{s})$ and cutting-edge angle of the tool $(30,45$ and 60 degrees $)$ on draft force as the dependent variable was evaluated.

The obtained results (Table 2) were analysed based on the factorial experiment using analysis of variance. Fig. 4 shows the comparison of the mean draft force (in $[\mathrm{N}]$ ) exerted on model tillage tool at various combinations of the four factors, tillage depth, speed, rake angle and cutting-edge angle. The plots on the diagonals show the overall level means while the offdiagonal shows the means for each pair of factor levels.

Based on the analysis made and because representation of the mean values is showed on the same vertical scale, it is very intuitive in showing the higher influence of the tool depth and rake angle and the little influence of the other two factors.

In order to obtain precise values of the influence shown, the estimated effects and interactions of the factors involved in experiment have been determined along with confidence intervals $(95 \%)$ and variance inflation factor. 
Table 2. Draft force variation in the experiment.

\begin{tabular}{|c|c|c|c|c|c|c|c|c|c|c|c|}
\hline \multirow{4}{*}{$\begin{array}{c}\text { D: Cutting } \\
\text { edge angle } \\
\text { [deg.] }\end{array}$} & \multirow{4}{*}{$\begin{array}{c}\text { C: Rake } \\
\text { angle } \\
\text { [deg.] }\end{array}$} & \multirow{4}{*}{$\begin{array}{c}\text { B: Tool } \\
\text { depth } \\
{[\mathrm{m}]}\end{array}$} & \multicolumn{9}{|c|}{ Draft force $[\mathrm{N}]$} \\
\hline & & & \multicolumn{3}{|c|}{ Run 1} & \multicolumn{3}{|c|}{ Run 2} & \multicolumn{3}{|c|}{ Run 3} \\
\hline & & & \multicolumn{3}{|c|}{ A: Tool speed $[\mathrm{m} / \mathrm{s}]$} & \multicolumn{3}{|c|}{ A: Tool speed $[\mathrm{m} / \mathrm{s}]$} & \multicolumn{3}{|c|}{ A: Tool speed $[\mathrm{m} / \mathrm{s}]$} \\
\hline & & & 0.67 & 0.98 & 1.39 & 0.67 & 0.98 & 1.39 & 0.67 & 0.98 & 1.39 \\
\hline \multirow{9}{*}{30} & \multirow{3}{*}{25} & 0.15 & \begin{tabular}{l|l|}
77.2 \\
\end{tabular} & 78.9 & 84.4 & 102.6 & 104.9 & 108.3 & 102.0 & 103.9 & 109.7 \\
\hline & & 0.2 & 161.1 & 162.6 & 167.3 & 206.6 & 209.1 & 214.3 & 206.7 & 207.8 & 213.5 \\
\hline & & 0.25 & 275.0 & 280.9 & 272.1 & 339.4 & 344.4 & 352.7 & 326.2 & 335.6 & 345.3 \\
\hline & \multirow{3}{*}{35} & 0.15 & 170.1 & 174.1 & 181.9 & 166.2 & 169.9 & 177.7 & 168.3 & 172.4 & 178.4 \\
\hline & & 0.2 & 308.5 & 312.4 & 321.0 & 311.2 & 315.2 & 323.4 & 309.2 & 313.4 & 321.5 \\
\hline & & 0.25 & \begin{tabular}{l|l}
475.1 \\
\end{tabular} & 494.9 & 510.2 & \begin{tabular}{l|l}
480.7 \\
\end{tabular} & 494.0 & 509.7 & 478.5 & 491.1 & 510.1 \\
\hline & \multirow{3}{*}{50} & 0.15 & 309.6 & 312.0 & 328.9 & 301.3 & 305.0 & 317.4 & 262.7 & 267.2 & 279.0 \\
\hline & & 0.2 & 571.3 & 582.8 & 588.9 & 543.1 & 549.1 & 557.2 & 539.7 & 546.7 & 556.4 \\
\hline & & 0.25 & \begin{tabular}{|l|}
807.7 \\
\end{tabular} & 841.7 & 874.1 & 757.4 & 793.8 & 820.4 & 795.0 & 807.1 & 845.2 \\
\hline \multirow{9}{*}{45} & \multirow{3}{*}{25} & 0.15 & \begin{tabular}{|l|}
98.7 \\
\end{tabular} & 100.2 & 105.7 & \begin{tabular}{l|l|}
99.8 \\
\end{tabular} & 101.4 & 107.2 & 99.8 & 102.2 & 107.9 \\
\hline & & 0.2 & 199.5 & 200.3 & 206.6 & 208.5 & 209.9 & 214.7 & 207.9 & 209.3 & 214.7 \\
\hline & & 0.25 & 335.0 & 339.3 & 345.0 & 340.4 & 344.4 & 350.7 & 325.6 & 335.4 & 345.6 \\
\hline & \multirow{3}{*}{35} & 0.15 & 167.8 & 171.0 & 177.9 & \begin{tabular}{l|l}
167.9 \\
\end{tabular} & 172.0 & 179.0 & 166.6 & 168.5 & 175.7 \\
\hline & & 0.2 & 312.9 & 321.6 & 328.9 & 313.8 & 317.6 & 325.2 & 310.8 & 313.1 & 323.5 \\
\hline & & 0.25 & \begin{tabular}{|l|l|}
499.7 \\
\end{tabular} & 513.7 & 526.8 & \begin{tabular}{|l|l|}
496.3 \\
\end{tabular} & 511.4 & 527.2 & 488.0 & 503.4 & 526.7 \\
\hline & \multirow{3}{*}{50} & 0.15 & 309.9 & 313.1 & 322.8 & 307.6 & 314.9 & 327.8 & 312.0 & 313.2 & 328.5 \\
\hline & & 0.2 & \begin{tabular}{|l|l|}
554.7 \\
\end{tabular} & 557.5 & 569.1 & \begin{tabular}{|l|l|}
556.3 \\
\end{tabular} & 566.0 & 581.9 & 561.9 & 568.8 & 581.2 \\
\hline & & 0.25 & 813.4 & 833.6 & 851.5 & 784.6 & 807.7 & 845.8 & 781.1 & 799.0 & 833.1 \\
\hline \multirow{9}{*}{60} & \multirow{3}{*}{25} & 0.15 & 85.5 & 86.3 & 92.6 & 94.8 & 93.8 & 95.3 & 104.4 & 105.6 & 111.3 \\
\hline & & 0.2 & 202.5 & 202.8 & 208.1 & 217.7 & 217.2 & 222.4 & 214.8 & 215.5 & 220.0 \\
\hline & & 0.25 & 337.3 & 342.1 & 353.4 & 343.2 & 352.0 & 360.0 & 340.2 & 347.1 & 357.9 \\
\hline & \multirow{3}{*}{35} & 0.15 & 153.9 & 156.2 & 161.6 & 174.6 & 176.9 & 185.4 & 173.9 & 177.1 & 184.7 \\
\hline & & 0.2 & 314.2 & 315.9 & 323.3 & 326.4 & 329.9 & 336.6 & 321.0 & 322.9 & 330.5 \\
\hline & & 0.25 & \begin{tabular}{|l|l|}
485.2 \\
\end{tabular} & 499.4 & 519.9 & 510.4 & 525.9 & 543.4 & 502.0 & 516.4 & 535.8 \\
\hline & \multirow{3}{*}{50} & 0.15 & 315.9 & 319.5 & 329.0 & 239.4 & 242.9 & 253.5 & 242.3 & 245.0 & 255.1 \\
\hline & & 0.2 & \begin{tabular}{|l|}
560.7 \\
\end{tabular} & 565.9 & 578.4 & 433.1 & 440.3 & 452.9 & 419.5 & 427.7 & 440.6 \\
\hline & & 0.25 & 800.2 & 831.3 & 862.5 & O.L. & O.L. & O.L. & O.L. & O.L. & O.L. \\
\hline
\end{tabular}

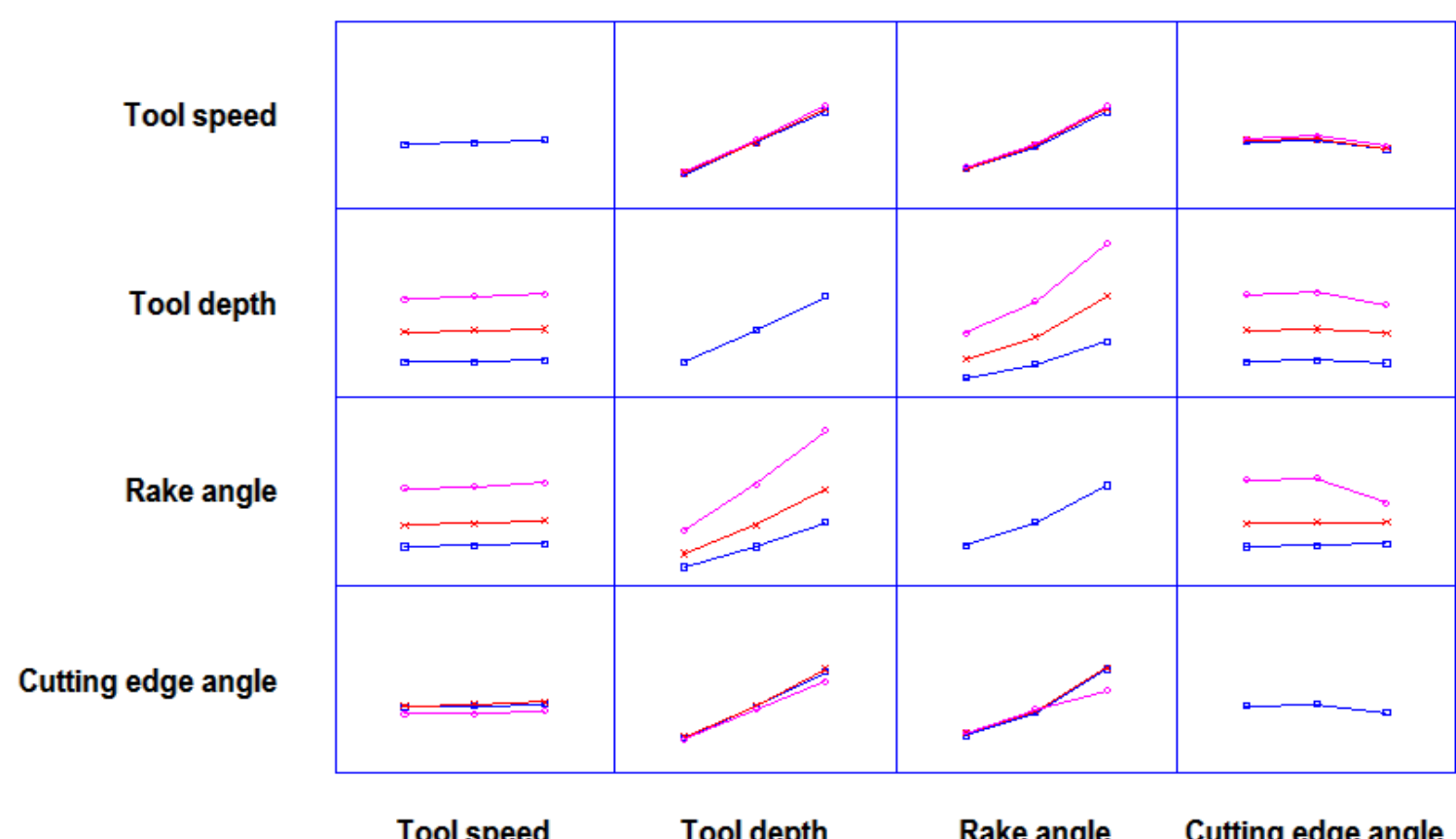

Fig. 4. Factor means plot for draft force. Vertical scale is set for 0 to 1000 [N]. 
Because the last six values in run 2 and 3, are missing from Table 3 (exerted values on the force sensor were higher than admissible limit), we didn't obtain a perfectly orthogonal design, this way the largest variance inflation factor was 1.35 .

The estimates are plotted in fig. 5 in a Pareto chart in decreasing order of their importance. Several less significant effects from statistical point of view have been removed from the chart. The data presented in this chart indicate the significant influence of tillage depth, penetration angle and forward speed on the draft force of the examined tillage tool model as well as BC and CD factor pair interaction. From the chart can be seen the positive effect of tool sharpening angle denoted by factor $\mathrm{D}$ in lowering the draft force.

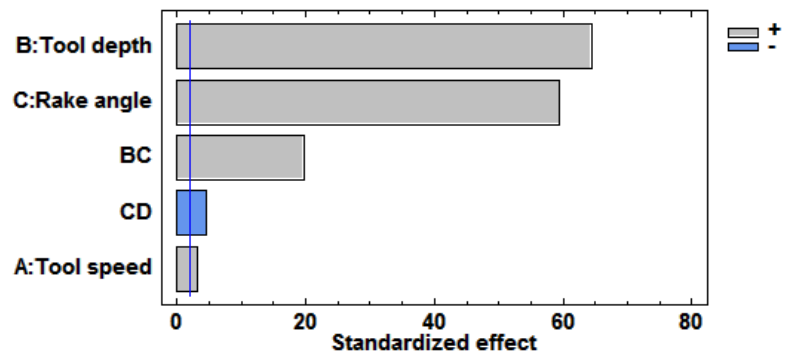

Fig. 5. Standardized Pareto chart for draft force.

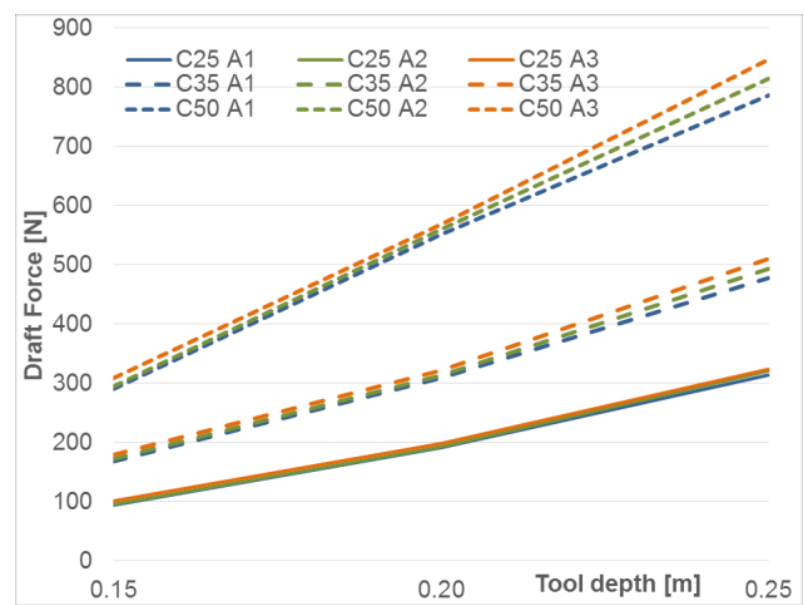

Considering that usually tillage depth is an imposed agro-technical condition of soil bed preparation and the fact that tool cutting edge angle is already establish by the manufacturer of tillage tools implements, a closer look to the speed and rake angle (parameters that usually are adjusted by the user) could offer a better understanding of the draft force variation. For that, in Fig. 6 the effect of depth, rake angle and tool speed under the influence of tool sharpening angle is presented. Draft force variation suggests a small and similar influence for both cases (represented in the figure) from the factor speed. As in figure 4, increasing the depth, the draft force increases as well almost proportional. Within the two cases represented (30 degrees versus 60 degrees tool sharpening angle) the combinations of factors, in this case the rake angle and cutting-edge angle have a significant influence in decreasing the draft force.

Unfortunately, from a statistical point of view, for this experiment, alone, the cutting-edge angle factor does not exhibit a significant influence over the draft force (the factor is missing from the Pareto chart). In our opinion, this fact can be attributed to the sand as testing medium (lack of cohesion and structure) and an unworn cutting edge.

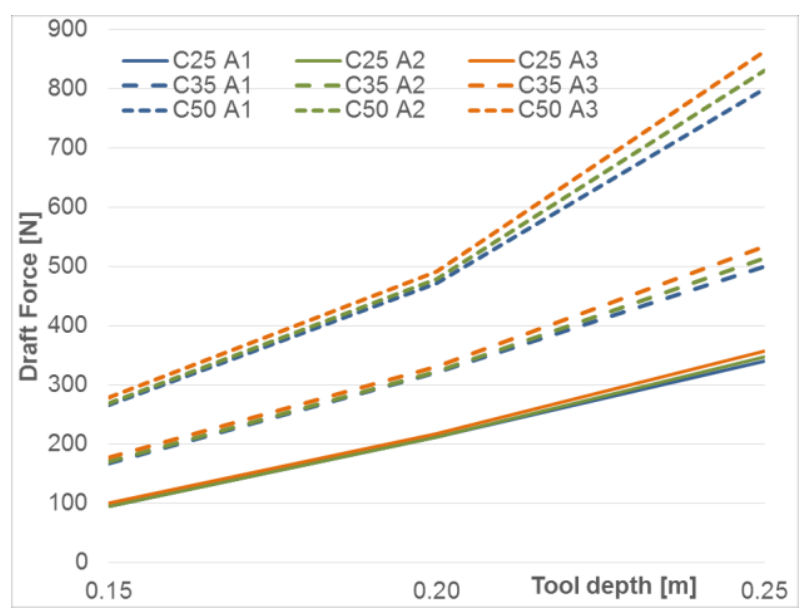

Fig. 6. Influence of tool depth, rake angle and speed over draft force at a cutting edge angle of $30^{\circ}$ and $60^{\circ}$.
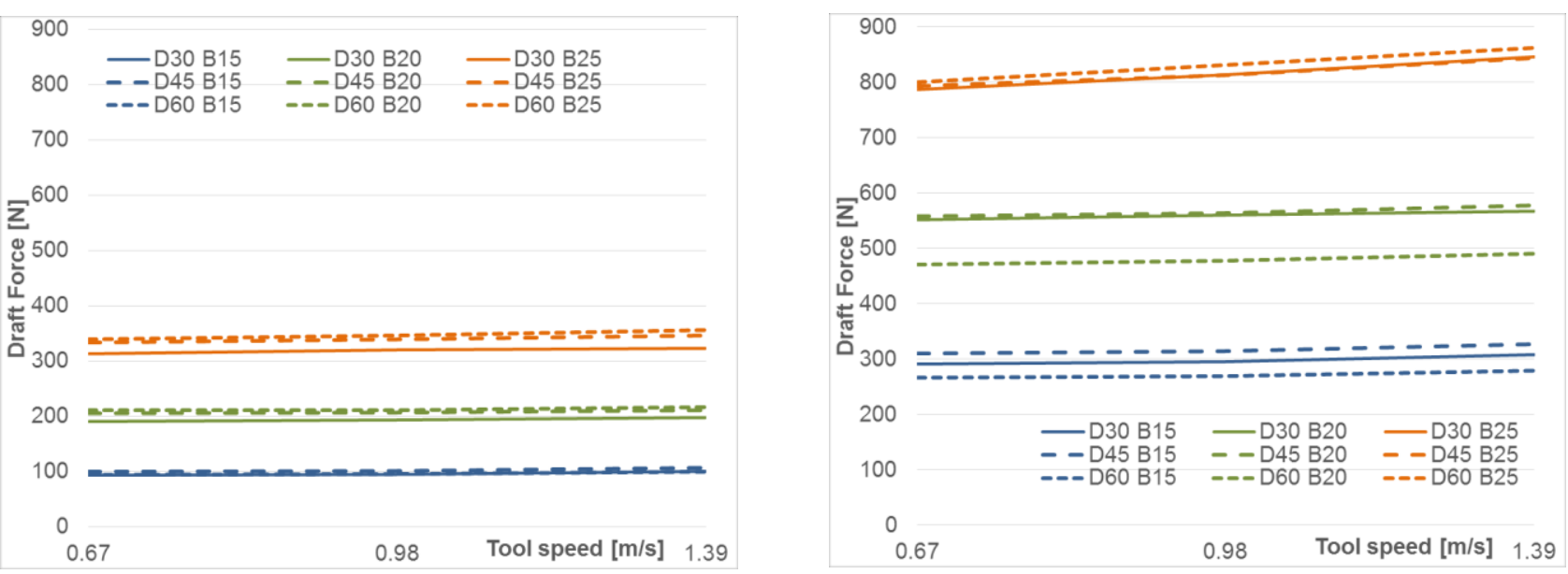

Fig. 7. Influence of speed, tool depth and cutting-edge angle over draft force at a rake angle of $25^{\circ}$ and $50^{\circ}$. 
Table 3. Analysis of Variance for Draft Force.

\begin{tabular}{|c|c|c|c|c|c|c|}
\hline Source & Sum of Squares & D.f. & Mean Square & F-Ratio & P-Value & \multirow{4}{*}{$\begin{array}{l}\mathrm{R}^{2}=97.20 \% \\
\mathrm{R}^{2}(\text { adjusted for d.f. })=97.06 \%\end{array}$} \\
\hline A: Tool speed & 13608.4 & 1 & 13608.4 & 11.37 & 0.0009 & \\
\hline B: Tool depth & 4.9965E6 & 1 & $4.9965 \mathrm{E} 6$ & 4174.69 & 0.0000 & \\
\hline C: Rake angle & $4.24147 \mathrm{E} 6$ & 1 & $4.24147 \mathrm{E} 6$ & 3543.84 & 0.0000 & \\
\hline D: Cutting edge angle & 1699.74 & 1 & 1699.74 & 1.42 & 0.2346 & \multirow{2}{*}{ Standard Error of Est. $=34.60$} \\
\hline $\mathrm{AB}$ & 3127.86 & 1 & 3127.86 & 2.61 & 0.1074 & \\
\hline $\mathrm{AC}$ & 2631.76 & 1 & 2631.76 & 2.20 & 0.1395 & \multirow{2}{*}{ Mean absolute error $=28.49$} \\
\hline $\mathrm{AD}$ & 12.5276 & 1 & 12.5276 & 0.01 & 0.9186 & \\
\hline $\mathrm{BC}$ & 466422. & 1 & 466422. & 389.71 & 0.0000 & \multirow{3}{*}{$\begin{array}{l}\text { Durbin-Watson statistic }=0.38 \\
(\mathrm{P}=0.0000)\end{array}$} \\
\hline $\mathrm{BD}$ & 1624.1 & 1 & 1624.1 & 1.36 & 0.2453 & \\
\hline $\mathrm{CD}$ & \begin{tabular}{|l|l|}
23761.9 \\
\end{tabular} & 1 & 23761.9 & 19.85 & 0.0000 & \\
\hline blocks & 2351.39 & 2 & 1175.7 & 0.98 & 0.3760 & \multirow{3}{*}{$\begin{array}{l}\text { Lag } 1 \text { residual autocorrelation }= \\
0.80\end{array}$} \\
\hline Total error & 268096. & 224 & 1196.86 & & & \\
\hline Total (corr.) & $9.58545 \mathrm{E} 6$ & 236 & & & & \\
\hline
\end{tabular}

A further investigation of the effect previous mentioned, by representing, in Fig, 7 the variation of draft force in relation to speed, depth and rake angle show in a similar manner the small effect of speed in increasing the draft force. Also, the increase of tillage depth will significant increase the draft force. But, comparing the two cases for which the rake angle takes 25 degrees and 50 degrees as values, the decrease of draft force is noticeable, again suggesting that a local minimum of draft force variable can be found with a proper combination of investigated factors.

In order to test the statistical significance of the effects an ANOVA analysis was performed and the results are presented in Table 3.

The ANOVA table partitions the variability in Draft Force into separate pieces for each of the effects. It then tests the statistical significance of each effect by comparing the mean square against an estimate of the experimental error.

In this case, five effects have P-values less than 0.05, indicating that they are significantly different from zero at the $95.0 \%$ confidence level. The R-Squared statistic indicates that the model as fitted explains $97.2 \%$ of the variability in Draft Force. The adjusted R-squared statistic, which is more suitable for comparing models with different numbers of independent variables, is $97.1 \%$. The standard error of the estimate shows the standard deviation of the residuals to be 34.5956. The mean absolute error (MAE) of 28.4902 is the average value of the residuals. The Durbin-Watson (DW) statistic tests the residuals to determine if there is any significant correlation based on the order in which they occur in your data file. Since the P-value is less than $5.0 \%$, that can be an indication of possible serial correlation at the $5.0 \%$ significance level. By plotting the residuals versus row order, we didn't observe a visible pattern, which eliminate the previous assumption.

Next step was to determine the mathematical formula that relate the studied factors at a convenient confidence model. For that, another set of statistical procedure was applied in the form of fitting a multiple linear regression model to describe as accurate as possible the relationship between dependant variable - draft force - and the four independent variables studied. For that, the regression models were sorted out based on several criteria (see Table 4): -models with largest adjusted R-squared; measures the proportion of the variability in Draft Force which is explained by the model. Larger values of adjusted RSquared correspond to smaller values of the mean squared error (MSE);

-models with best information criteria based on Akaike Information Criterion (AIC) with the goal of selecting a model with small residual error and as few coefficients as possible; The best model is the one that minimizes the information criterion;

-models with smallest values of Mallows' $C_{p}$ statistic who measure the bias in a model, based on a comparison of total mean squared error to the true error variance.

Table 4. Selected criteria-based models.

\begin{tabular}{|c|c|l|l|l|c|}
\hline MSE & Coef. & Adj. $\mathbf{R}^{\mathbf{2}}$ & AIC & $\mathbf{C}_{\mathbf{p}}$ & Included variables \\
\hline 3391.65 & 5 & 91.65 & 8.17 & 5.00 & $\mathrm{ABCD}$ \\
\hline 3398.58 & 4 & 91.63 & 8.16 & 4.48 & $\mathrm{ABC}$ \\
\hline 3439.20 & 3 & 91.53 & 8.17 & 6.28 & $\mathrm{BC}$ \\
\hline
\end{tabular}

For the three selected models, the ordinary least squares procedure of multiple linear regression model was applied with and without Cox-Box optimization (Table 5). The R-Squared statistic values indicates that the models used as fitted explains in the specific percentage the variability in Draft Force dependant variable. The standard error of the estimate (not presented in the table) for each model can be used to construct prediction limits for new observations.

Table 5. Selected regression fitting procedure.

\begin{tabular}{|c|c|c|}
\hline \multirow{2}{*}{ Variables } & Ordinary Least Squares & Box-Cox optimization \\
\cline { 2 - 3 } & $\mathbf{R}^{\mathbf{2}}[\mathbf{\%}]$ & $\mathbf{R}^{\mathbf{2}}[\mathbf{\%}]$ \\
\hline $\mathrm{ABCD}$ & 91.79 & $98.15-$ rel. (1) \\
\hline $\mathrm{ABC}$ & 91.74 & 98.15 \\
\hline $\mathrm{BC}$ & $91.60-$ rel. (2) & 98.03 \\
\hline
\end{tabular}

From the model used, two outputs are further presented, respectively the most complete regression model (rel. 1) and the simplest model (rel. 2). Within the specified limits and constrains of the experiment the second mathematical relation explains with enough accuracy the variability of the dependant variable according to AIC criteria. 


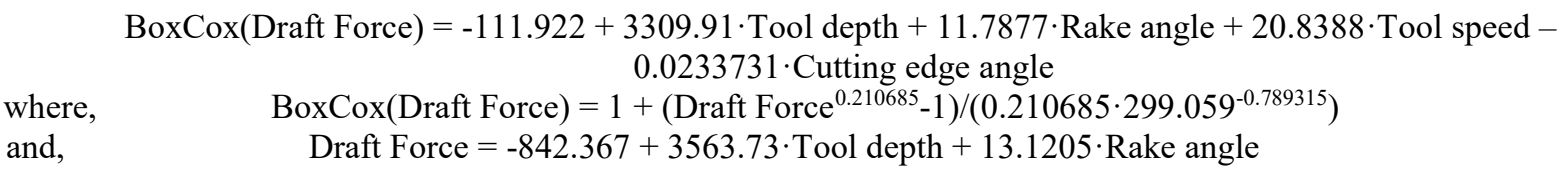

Using the Box-Cox optimization procedure, the output shows the results of fitting a multiple linear regression model to describe the relationship between Draft Force and the selected independent variables. The equation of the fitted model is presented in relation (1).

As previously mentioned, in table 6, the simplest model obtained presented in relation (2) can explain $91.60 \%$ of the variability in draft force variable.

Either of the two mathematical relation, within the specified limitations, can be successfully used in predicting the behaviour of the draft force in the specified model. More than that, at this level of understanding of the soil-tool independent variables interaction, considering that several agro-technical parameters are imposed in the cultivation process, the mathematical relations can be used to optimize the draft force.

\section{Conclusions}

A soil-tool interaction model was investigated in an indoor controlled conditions soil-bin experiment.

The tools used were simple in geometry in order to reduce the complexity model for a better understanding of the variables interaction.

The measured results were intentionally presented in a tabular form to be compared with similar experiments, computer simulations or analytical soil-tool interaction models. In the present investigation, the tool depth and the rake angle were determined to have, individually taken, each one more than $60 \%$ influence in the variability of tool draft force with an increase effect up to $30 \%$ in the case on combining the two. The other two investigated variables have a smaller effect over the dependent investigated variable.

The most interesting observation is the positive effect of decreasing the draft force encountered in the case of modifying the cutting-edge angle, the tool sharpening angle, in the most unfavourable situations for tillage tools operation respectively at higher rake angle, depth or tool speed.

Further investigation is needed especially at higher speed and with different geometries.

This work was supported by Grant of the Romanian National Authority for Scientific Research, CNCS, UEFISCDI, PN-IIIP2-2.1-BG-2016, Project number: 78BG/2016, SC Artecom SRL upgraded competences through deep soil tillage machinery optimization.

\section{References}

1. Ibrahmi, H. Bentaher, E. Hamza, A. Maalej, A.M. Mouazen, Computers and Electronics in Agriculture 117, 268-275 (2015)

2. R.J. Godwin, Soil \& Tillage Research 97, 331340 (2007)

3. M.I. Ghereş, INMATEH - Agricultural Engineering 42 (1), 5-12 (2014)

4. M.H. Abbaspour-Fard, S.A. Hoseini, M.H. Aghkhani, A. Sharifi, Span. J. Agric. Res., 12 (1), 44-51 (2014)

5. M. Askari, S. Khalifahamzehghasem, WASJ 21 (12), 1789-1794 (2013)

6. S.I. Manuwa, Soil \& Tillage Research 103, 399405 (2009)

7. O.S. Ogbeche, M.S. Idowu, Agric Eng Int: CIGR Journal 18 (1), 91-101 (2016)

8. A. Ani Ozoemena, B.B. Uzoejinwa, A.O. Ezeama, A.P. Onwualub, S.N. Ugwua, C.J. Ohagwua, Soil \& Tillage Research 175, 13-27 (2018)

9. E. M. Nagy, L. V. Fechete, C. Coţa, S. Stănilă, D. Herak, INMATEH - Agricultural Engineering 32 (3), 5-12 (2010)

10. L. Vlădutoiu, T. Andrei, L. Fechete, E. Marin, V. Vlădut,, M. Matache, I. Dumitru, Annals of the University of Craiova - Agriculture, Montanology, Cadastre Series 45 (2), 253-258 (2015)

11. S. Karmakar, S.R. Ashrafizadeh, R.L. Kushwaha, Journal of Terramechanics, 46, 277-283 (2009)

12. M. Topakci, H. K. Celik,, M. Canakci, A. E. W. Rennie, I. Akinci, D. Karayel, JFAE 8 (2), 531 $536(2010)$

13. T. Marakoglu, K. Carman, Journal of Agronomy 8 (1), 21-26 (2009)

14. A. Moeenifar, S. R. Mousavi-Seyedi, D. Kalantari, Agric Eng Int: CIGR Journal 16 (1), 6974 (2014)

15. L. Naderloo, R. Alimadani, A. Akram, P. Javadikia, H. Zeinali Khanghah, JFAE 7 (3-4), 382385 (2009)

16. R.J. Godwin, M.J. O'Dogherty, Journal of Terramechanics, 44, 3-14 (2007) 